\title{
High-density lipoprotein (HDL) cholesterol - more complicated than we think?
}

\author{
Katarzyna Nessler ${ }^{1, A-B, D-F}$, Adam Windak ${ }^{1, A, D-F}$, Rafał Grzybczak ${ }^{2, A, C-E}$, Michał Bohdan Nessler $^{3, A, C-D}$, \\ Aleksander Siniarski ${ }^{4, A, D}$, Grzegorz Gajos ${ }^{4, A, D-F}$ \\ ${ }^{1}$ Department of Family Medicine, Chair of Internal Medicine and Gerontology, Jagiellonian University Medical College, \\ Krakow, Poland \\ ${ }^{2}$ Department of Cardiac Rehabilitation, Institute of Cardiology, Jagiellonian University Medical College, Krakow, Poland \\ ${ }^{3}$ Burns and Plastic Surgery Centre of Malopolska, Rydygier Memorial Hospital, Krakow, Poland \\ ${ }^{4}$ Department of Coronary Disease and Heart Failure, Institute of Cardiology, Jagiellonian University Medical College, \\ John Paul II Hospital, Krakow, Poland \\ A - Research concept and design, B - Collection and/or assembly of data, C - Data analysis and interpretation, \\ $D$ - Writing the article, E - Critical revision of the article, F - Final approval of article
}

Nessler K, Windak A, Grzybczak R, Nessler MB, Siniarski A, Gajos G. High-density lipoprotein (HDL) cholesterol - more complicated than we think? Ann Agric Environ Med. 2018; 25(3): 517-526. doi: 10.26444/aaem/92350

\begin{abstract}
Introduction and objective. There are some clinical situations where a high level of HDL cholesterol (HDL-C) may be unfavourable. In these situations, HDL-C may undergo some changes, and even if its quantity is within the reference range, its quality is no longer the same.

Brief description of state of knowledge. Diabetes is the state of elevated oxidative stress. Studies conducted to-date have revealed an increased production of the reactive forms of oxygen as the result of tissue damage in diabetes patients. The expression 'dysfunctional HDL' has been coined in the literature to describe high-density lipoproteins that lose their antioxidative and anti-inflammatory properties, that is, $\mathrm{HDL}-\mathrm{C}$ that loses its basic functions. Recent observational studies have confirmed that the atheroprotective activity of properly functioning HDL-C is frequently impaired in clinical situations associated with oxidative stress. The presented review lays the foundation for a new approach to understanding how the functional properties of HDL help reduce cardiovascular risk.

Conclusions. In the light of presented findings it seems that there is a need to seek a better diagnostic marker than HDL-C level. This study presents some possible directions for future research to bring us closer to the full understanding of the HDL particle and its role in patients with ischemic heart disease and type 2 diabetes.
\end{abstract}

\section{Key words}

cardiovascular disease, diabetes mellitus, dysfunctional HDL

\section{INTRODUCTION}

The incidence of diabetes continues to rise worldwide, with the number of patients exceeding 425 million adults and expected to rise to 642 million people by 2040 [1]. This growing incidence has been related to urbanization and dramatic lifestyle changes, particularly in developed countries. As a result, there has been an increase in the incidence of risk factors for numerous diseases, including type 2 diabetes [2]. So far, several modifiable risk factors have been associated with type 2 diabetes, including overweight, unhealthy diet, and lack of physical activity [2]. The most recent evidence shows that the growing incidence of diabetes constitutes a huge health burden for global society [2].

The major cause of mortality and morbidity in diabetic patients is cardiovascular disease (CVD) [3]. Moreover, in patients with diabetes and concomitant CVD, the risk of major adverse cardiac events is increased, compared with patients without diabetes [3]. On average, it is estimated that patients with type 2 diabetes die prematurely, about 5-10 years earlier than those without type 2 diabetes, mostly due

Address for correspondence: Katarzyna Nessler, Department of Family Medicine, Chair of Internal Medicine and Gerontology, Jagiellonian University Medical College in Krakow, 4 Bochenska str, 31-061 Krakow, Poland

e-mail: katarzynanessler@gmail.com

Received: 24.03.2018; accepted: 17.06.2018; first published: 28.06.2018 to coronary heart disease (CHD) [4]. In Europe, the treatment of CVD accounts for a significant proportion of health care costs attributed to type 2 diabetes $(10 \%-12 \%)$ [1].

Dyslipidaemia is the major risk factor for atherosclerosis, although to-date the mechanism of this association has not been fully elucidated. Low-density lipoprotein cholesterol (LDL-C) has been determined as the major atherogenic lipoprotein, and its central role in atherosclerosis has been confirmed in numerous studies [5-7]. On the contrary, epidemiological, pathological, and experimental studies have demonstrated that high-density lipoprotein cholesterol (HDL-C) may protect against coronary artery disease [8, 9]. Moreover, HDL-C has been shown to reduce the risk of atherosclerosis by multiple pathophysiological mechanisms.

Despite the atheroprotective properties of HDL-C, some most recent clinical studies have identified individuals with a significant atherosclerotic burden despite normal or elevated levels of HDL cholesterol [8]. Nascent discoidal HDL-C is composed of cholesterol, apolipoprotein A-I (apoA-I) and phospholipids. Its particles undergo lipidation and remodeling by a series of reactions mediated by ATP-binding cassette sub-family G member 1 (ABCG1), membrane-associated ATP-binding cassette transporter A1 (ABCA1), hepatic lipase, endothelial lipase, cholesteryl ester transfer protein (CETP), and phospholipid transfer protein $[10,11]$. This complex structure and involvement 
in various metabolic pathways are the potential causes of qualitative and quantitative modifications of HDL particles, which may result in the loss of at least a few pleiotropic properties of HDL-C and the development of new potentially proatherogenic activities. Therefore, the HDL-C level itself is not always a good indicator of its atheroprotective features.

Since it has been established that a significant number of CHD events in patients with type 2 diabetes occur in individuals with normal or even elevated HDL-C level, it is necessary to identify biomarkers with a better predictive value in this group of patients. In addition, these patients have atherogenic dyslipidemia and therefore may develop atherosclerosis.

The mechanisms that deprive HDL-C of its cardioprotective properties are poorly understood. Therefore, the presented review aimed to discuss some of the possible directions for future research to bring us closer to a full understanding of the HDL particle and its role in patients with ischemic heart disease and type 2 diabetes.

Biocharacteristics of HDL-C. The HDL particle consists of lipids and proteins (apolipoproteins) with different biochemical activities. So far, over 100 proteins bound to HDLs have been identified, with apoA-I as the main surface protein. Immunoseparation is used to separate HDL particles containing apoA-I from those containing both apoA-I and apolipoprotein A-II (apoA-II) (Fig. 1 and 2).

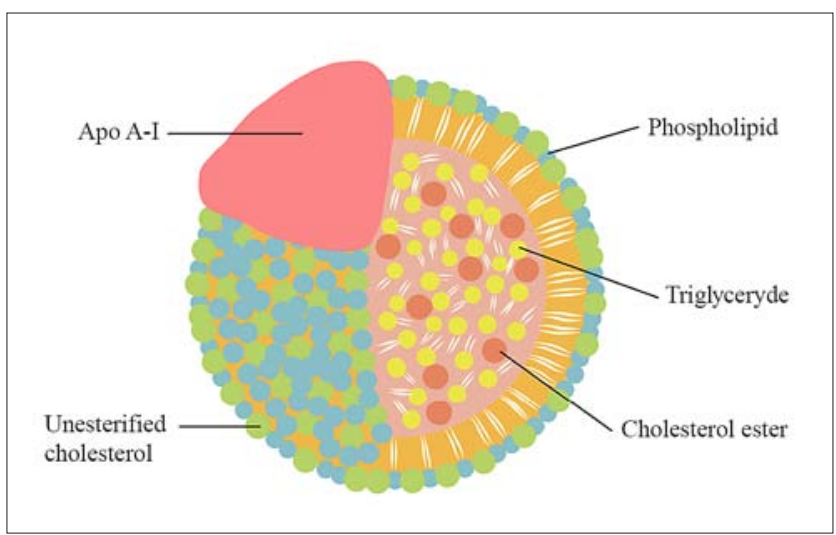

Figure 1. HDL particle containing apoA-I

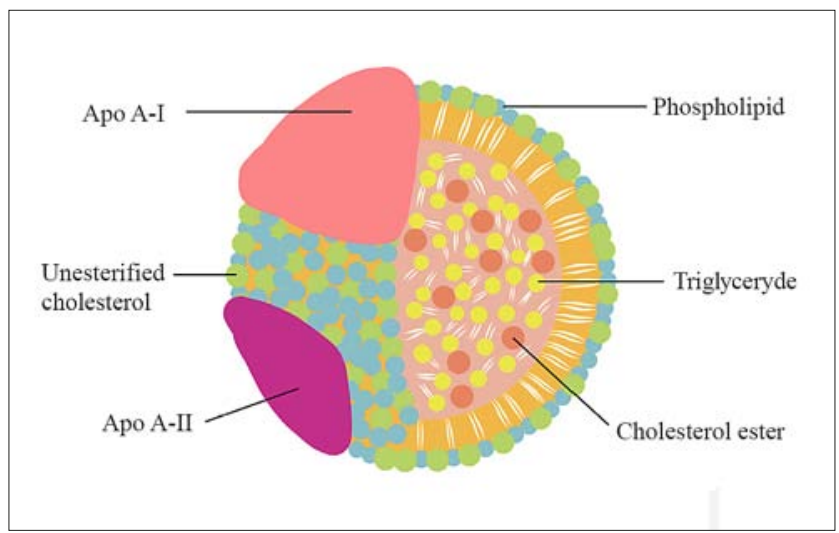

Figure 2. HDL particle containing ApoA-I-A-II

It has been suggested that these apolipoproteins have different metabolic properties and thus may have a different atheroprotective potential [12]. This cited experimental study revealed reduced anti-inflammatory activity in transgenic mice with the over-expression of apoA-II in HDL particles. The authors also hypothesized that patients with ischemic heart disease and type 2 diabetes may show differences in HDL protein profiles.

Interestingly, little is known about proteins other than apoA-I contained in normal and in dysfunctional HDL-C, and data on the potential role of different HDL-C subpopulations in the inflammatory process are still limited [13]. Meanwhile, a growing body of evidence indicates that the determination of HDL subpopulations might significantly improve our knowledge on cardiovascular risk [14]. It is believed that such data would be particularly valuable for diabetic patients.

HDL-C as a protective factor. HDL-C is currently being widely studied in experimental and clinical research. It has been recognized as an independent protective factor in CHD. This was first shown in the Framingham Heart Study, which confirmed an inverse correlation between HDL-C and the incidence of CHD [15]. HDL-C exerts anti-inflammatory and antioxidant effects and protects against atherosclerosis, as shown by a considerable amount of experimental data [16]. There are many recognized pathways that have been proposed to account for the involvement of HDL-C in protective mechanisms against $\mathrm{CHD}$.

HDL-C and its major protein, apoA-I, are essential for mediating reverse cholesterol transport, which is a multi-step process involving cholesterol transfer from peripheral tissues, such as arterial walls, back to the liver via plasma for excretion. The HDL transformation cycle is shown in Figure 3.

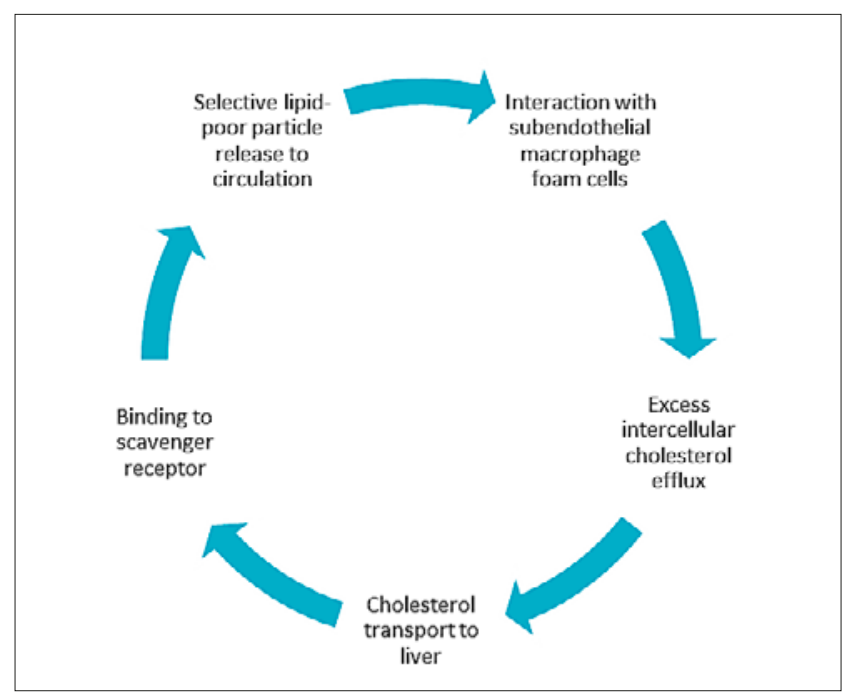

Figure 3. HDL-C transformation cycle

This function likely plays an important role in the atheroprotective mechanism of HDL-C. Other protective activities of HDL-C include the removal or detoxication of oxidized sterols and phospholipids. It also exerts antithrombotic actions and has a beneficial effect on endothelial cells $[17,18]$ (Tab. 1).

Effects of a pharmacological increase in HDL levels. A meta-analysis of randomized controlled trials has not confirmed the hypothesis that any agent raising HDL level should decrease the number of cardiovascular events [19]. 
Table 1. Main features of HDL cholesterol

\begin{tabular}{|c|c|}
\hline Features of HDL cholesterol & Description \\
\hline $\begin{array}{l}\text { Anti-inflammatory and } \\
\text { antioxidant (detoxification } \\
\text { of oxidized sterols and } \\
\text { phospholipids) }\end{array}$ & $\begin{array}{l}\text { Protective role against atherosclerosis. Main antioxidant protein of HDL is Apo A-I. Its role strongly depends on myeloperoxidase, which } \\
\text { promotes endothelial dysfunction and plaque rupture. Excessive production of malondialdehyde and phospholipid aldehyde resulted } \\
\text { from the fact that oxidative stress deprives the ability of Apo A-I to promote atheroprotective features. On the other hand, paraoxanase-1 } \\
\text { in HDLs limits lipid oxidation and provides additional atheroprotective effects. }\end{array}$ \\
\hline $\begin{array}{l}\text { Reverse cholesterol } \\
\text { transport }\end{array}$ & $\begin{array}{l}\text { Protective role against atherosclerosis by transporting the cholesterol from arterial walls and peripheral tissues to the liver and activating } \\
\text { nitric oxide synthase. }\end{array}$ \\
\hline Antithrombotic activity & $\begin{array}{l}\text { The effect of HDL-C on platelet function remains unclear. Some studies showed that HDL particles changed platelet signaling pathways by } \\
\text { limiting intraplatelet cholesterol overload and inhibiting nitric oxide and prostacyclin production. Decreased HDL-C level was associated } \\
\text { with high platelet reactivity both in patients with ST-elevation myocardial infarction and stable coronary artery disease. }\end{array}$ \\
\hline
\end{tabular}

Healing the endothelium

HDL particles inhibit the VCAM-1 expression. The inhibition of this process was observed in diabetes and cardiovascular diseases and led to increased inflammatory activity by macrophage adhesion to activated endothelial cells.

LDL cholesterol (LDL-C)-lowering agents. The finding that elevated LDL-C and low HDL-C level is associated with increased cardiovascular mortality encouraged the search for targeted drug treatments. The main assumption for new therapies was either to increase HDL-C level or lower LDL-C level, or to achieve both. Since then, lowering of LDL-C level by using statins has repeatedly been found to reduce the risk of cardiac events and all-cause mortality in the setting of secondary and primary prevention [19].

Multiple strategies targeting the inhibition of proprotein convertase subtilisin/kexin type 9 (PCSK9) have emerged as effective modalities for LDL-C lowering. PCSK-9 monoclonal antibodies are the most advanced to-date in clinical development, and in 2015 alirocumab and evolocumab were approved for clinical use by regulatory agencies. Adverse events associated with these medications are minimal. Importantly, most studies confirmed that PCSK9 improve clinical CVD outcomes, although the results of a long-term study are yet to be reported [20].

Contrary to the above findings, the authors of the most recent study published in the European Heart Journal have found no associations between PCSK9 and apoA-I, HDL-C, lipoprotein(a) and high-sensitivity C-reactive protein. Furthermore, the baseline levels of PCSK9 did not predict the first cardiovascular events [21]. In addition, it should be noted that the more general use of PCSK-9 monoclonal antibodies has thus far been limited by the high cost [20].
Nowadays, with the generic availability of statins and their widespread use, the aim of reducing LDL level has been fulfilled. However, the achievement of the second goal, namely, elevating HDL level, seems to be more complicated.

HDL-C-elevating agents. The three main substances proposed to increase HDL level with the aim of reducing cardiovascular morbidity and mortality are niacin, fibrates, and the recently developed CETP inhibitors. Different classes of lipid-modifying agents are presented in Table 2.

A recent study, HPS2-Thrive, has reported that the addition of niacin to statin-based LDL-C-lowering therapy did not significantly reduce the risk of major vascular events, but it did increase the risk of serious adverse events (such as gastrointestinal complications, bleeding, musculoskeletal side effects, or increased incidence of diabetes) [22, 23].

Fibrates have been shown to consistently reduce some cardiovascular outcomes but only in patients with high serum triglyceride levels and low HDL-C level. Fibrates, unlike CETP, increase HDL-C level mostly through the stimulation of apoA-I production [24].

In the class of CETP inhibitors, three agents have been studied: anacetrapib, dalcetrapib, and torcetrapib. Two studies involving the promising torcetrapib were stopped prematurely because of adverse events in the treatment arms, and one study involving dalcetrapib was also discontinued because of futility [25-27]. Although the above CETP

Table 2. Lipid-modifying agents

\begin{tabular}{ll}
\hline Pharmacological targets & Description \\
\hline Statins & $\begin{array}{l}\text { Reduced cardiac events and all-cause mortality, mostly by lowering LDL cholesterol. The effect on HDL-C is more complicated } \\
\text { and less well-investigated. }\end{array}$ \\
\hline Niacin & $\begin{array}{l}\text { Adding niacin to statin did not significantly reduce the risk of cardiovascular disease and increased the risk of major side- } \\
\text { effects (e.g. gastrointestinal, bleeding, musculoskeletal) }\end{array}$ \\
\hline Fibrates & Fibrates increase HDL-C level mostly through the stimulation of apolipoprotein A-I production. \\
\hline $\begin{array}{l}\text { CETP inhibitors (anacetrapib, } \\
\text { dalcetrapib, and torcetrapib, TA-8995) }\end{array}$ & $\begin{array}{l}\text { Beneficial effects of TA-8995 on lipid profiles in patients with dyslipidaemia, well-tolerated by patients. No evidence of the } \\
\text { effectiveness of other agents, or trials discontinued due to adverse events. }\end{array}$ \\
\hline PCSK-9 monoclonal antibodies & $\begin{array}{l}\text { The FOURNIER study proved that it significantly reduces major vascular events in patients with stable atherosclerotic } \\
\text { cardiovascular disease, also patients with prior MI. Patients closer to their most recent MI, with multiple prior MIs or residual } \\
\text { multivessel CAD benefit from substantial risk reductions with evolocumab. }\end{array}$
\end{tabular}

Evolocumab

Evolocumab significantly reduces cardiovascular risk in patients with and without diabetes. 
inhibitors have not been able to prevent clinically important events, a number of clinical trials (such as ACCELERATE and REVEAL) with other CETP inhibitors are still ongoing, and the results are anticipated in the next few years.

The authors of a recent study published in The Lancet, TULIP, presented the results for a novel CETP inhibitor called TA-8995. This agent has been shown to have beneficial effects on lipid profile in patients with dyslipidemia and to be well-tolerated by patients. Moreover, it did not cause any serious adverse events [28]. In this randomized, double-blind, placebo-controlled study, HDL-C levels were increased in patients receiving TA-8995, with larger increases correlating with higher doses. Although the authors underlined that further studies were needed to determine the effect of TA8995 on cardiovascular outcomes, the results are promising.

Although the results of the TULIP study are encouraging, most of the attempts to reduce cardiovascular events or mortality by increasing HDL-C level using those three different classes of drugs on top of statins have been unsuccessful so far [29].

Insight from genetic studies. A large-scale meta-analysis of genome-wide association studies revealed that plasma lipid levels are affected by common genetic variants, which explains from $10 \%-12 \%$ of the total variance and from $25 \%-30 \%$ of genetic variability in plasma lipid phenotypes [30]. This means that although a portion of the genetic contribution to variation in plasma lipids and lipoproteins has been characterized, there is still variance that remains without known assignment [31].

The evidence from a cumulative meta-analysis and replication studies suggests that a more careful examination of the common variants is required, considering the available genetic data. This might help explain the portions of missing heritability, as well as clarify the pathways and mechanisms involved in lipid metabolism and CVD. The authors of a recent article published in the American Journal of Human Genetics suspect the possibility of identification of potential loci in which rare single nucleotide polymorphisms (SNPs) with large effects on the phenotype can be discovered [32]. To investigate the causal role of HDL-C and triglycerides in CVD using multiple instrumental variables for Mendelian randomization, Holmes et al. developed weighted allele scores based on SNPs with established associations with HDL-C, LDL-C, and triglycerides. The findings from this analysis (which included over 62,000 participants with 12,000 CVD events) support the causal effect of triglycerides on the risk of CVD, while the involvement of HDL-C has not been confirmed [33].

Should we aim at increasing HDL-C and apoA-I levels? It is still unclear whether an increase in HDL-C and apoA-I level is associated with a reduced risk of cardiovascular events. A recent study by Boekholdt et al. showed that an increase in HDL-C level was not associated with a lower risk of major cardiovascular events, independently of established risk factors. However, the authors reported an association between increased apoA-I level and a reduced risk of major cardiovascular events [34]. These results were consistent with the data reported by the EPIC-Norfolk and Rotterdam Studies, which suggested that the association between changes in HDL-C level caused by lipid-modifying therapy and risk of $\mathrm{CHD}$ was mostly explained by established risk factors [35]. Based on combined data from those studies, the change in HDL-C level caused by lipid-modifying therapy was associated with a reduced cardiovascular risk when adjusted for age, gender, and baseline HDL-C level. However, this association was attenuated and was not significant when further adjusted for non-HDL-C (calculated as total cholesterol level minus HDL-C level, as LDL-C and triglyceride level were not measured for all participants during all examination rounds), as well as for smoking history, prevalent diabetes, systolic blood pressure, body mass index, use of antihypertensive medications, previous myocardial infarction, prevalent angina, and previous stroke. These studies provided no evidence to support a significant benefit of increasing HDL-C level, independently of the effect of lowering non-HDL-C.

A systematic review and meta-regression analysis of randomized controlled trials testing lipid-modifying interventions showed that an increase in HDL-C level was not associated with cardiovascular outcomes [36]. The analysis included 108 randomized trials involving 299310 participants at risk of cardiovascular events. There was no association between treatment-induced rise in HDL-C level and risk ratios for CHD-related or total mortality and CHD events. A study performed by Grover et al. showed that the change in HDL-C level was a strong independent risk factor for CV events [37]. In contrast, a recent published meta-analysis showed that a rise in HDL-C level was not associated with a lower risk of major cardiovascular events independent of established risk factors [34]. However, an increase in apoA-I level was associated with cardiovascular risk such that patients with the largest increases had the lowest cardiovascular risk [34]. The authors argued that although these results did not provide evidence for the potential causality of the association between increased apoA-I level and reduced cardiovascular risk, they supported further research into apoA-I-modifying therapies.

In summary, most recent studies have questioned the hypothesis that lipid-modifying therapy should be aimed at increasing HDL-C level. On the other hand, there have been studies showing that an increase in apoA-I level is associated with a reduced cardiovascular risk, independently of established risk factors, which reinforces the rationale for using apoA-I as a target for the treatment of atherosclerosis.

Selected indices of HDL and their superiority over chemical measurement of HDL-C level. Recent studies of drugs that increase HDL-C level, but without reducing the rate of cardiovascular events or progression of atherosclerosis, revealed the limitations of the specific agents tested [3840]. HDL particles (HDL-P) are quite heterogeneous and because their activity cannot be inferred from the chemically measured plasma HDL-C level, some recent studies have focused on measuring other indices of HDL, such as the function, size, or concentration (number) of HDL-P. It is hypothesized that these indices may be better clinical markers of atheroprotective effects of HDL [41].

A review published in 2011 by Asztalos et al. confirmed that HDL subclasses differ in physical and chemical properties, protein and lipid composition, metabolism, and physiological functions, and, consequently, they have different pathophysiological significance [41]. The results from the large, randomized, double-blind JUPITER trial showed that among patients who did not receive lipidlowering therapy, HDL-C, apoA-I, and HDL-P level showed 
similar inverse associations with CVD [42]. However, among individuals who were treated with rosuvastatin, HDL-P had a stronger and statistically significant inverse association with CVD in comparison with HDL-C or apoA-I level. The authors suggested that HDL-P may be a better marker of residual risk than HDL-C or apoA-I among individuals receiving potent statin therapy to achieve very low LDL-C level. It has also been shown that the association of HDL-C level with CVD is influenced by insulin resistance, abdominal obesity, and inflammation [43]. In contrast, HDL-P appeared to be much less influenced by these factors [44].

So far, only HDL-C has been the standard parameter of HDL measured in clinical practice. However, it may be important to assess also other parameters in clinical trials of HDL-elevating therapies. Considering the results of the recent studies, it seems that HDL-P may be an alternative to HDL-C as a marker of HDL-related cardiovascular risk.

HDL-C efflux capacity. HDL-C exerts numerous antiatherosclerotic effects that are not readily reflected by HDL-C level [45]. The key function of HDL is to promote reverse cholesterol transport from peripheral tissues to the liver, and the critical initial step in this transport is cholesterol efflux from macrophages to HDL [46]. Cholesterol efflux by HDL is also required for lipoprotein signaling in endothelial cells, which underlies the ability of HDL to activate endothelial nitric oxide synthase, promote endothelial repair, and induce angiogenesis [47]. Impaired cholesterol efflux capacity has also been shown to correlate with increased platelet reactivity in vitro [48].

Macrophage-specific cholesterol efflux capacity has been directly and causally linked to the prevention of atherosclerosis in animal models [46]; therefore, it has become the focus of recent research as a promising indicator of HDL function with possible antiatherosclerotic effects [49]. Khera et al hypothesized that cholesterol efflux capacity is a determinant of atherosclerotic burden that was independent of HDL-C level [50]. The authors examined the relationship of efflux capacity with HDL-C level and two measures of atherosclerosis, namely, carotid intimamedia thickness and angiographically confirmed CHD. They showed that cholesterol efflux was inversely correlated with both measures, independently of HDL-C level. In addition, cholesterol efflux was associated with a higher level of apoA-I, also independently of HDL-C level. Moreover, a recent population-based cohort study involving individuals without CVD at baseline showed that cholesterol efflux capacity was inversely correlated with incident atherosclerotic CVD [51]. This association was also observed after adjustment for traditional cardiovascular risk factors, HDL-C level, and number of HDL-P. Rohatgi et al. suggested that the association of HDL function with cardiovascular risk is explained by processes other than those shown by the HDL-C level, HDL-P concentration, or traditional cardiovascular risk factors [51]. Finally, Hutchins et al. recently reviewed evidence that cholesterol efflux capacity is a strong inverse predictor of incident and prevalent CVD. The authors suggested that impaired macrophage cholesterol efflux to HDL contributes to the risk of CVD [52].

It should be noted that cholesterol efflux from macrophages represents only a small fraction of the overall flux through the reverse-cholesterol-transport pathway. However, this might be the most relevant component in atheroprotection [53].
HDL and type 2 diabetes. In glucose-resistant tissue observed in such conditions as metabolic syndrome, nonenzymatic glycosylation of HDL particles was shown to occur, which was considered as one of the main mechanisms of dysfunctional HDL. This modification significantly impairs the reverse cholesterol transport from peripheral tissues via ABCA1 and ABCG1 transporters. Glycated substrate molecules are also susceptible to oxidation and lose antioxidant properties themselves. In addition, they show a dramatically lower potential to inhibit the release of proinflammatory cytokines (tumour necrosis factor alpha and interleukin $1 \mathrm{~b}$ ) from activated macrophages. Also, the glycosylation process reduces the HDL-mediated activation of lecithin-cholesterol acyltransferase, an enzyme that catalyzes the conversion of cholesterol to cholesteryl ester. It has been shown that without this conversion, mature spherical HDL particles are not formed and excess peripheral tissue cholesterol is less efficiently transported to the liver for excretion from the body [54].

The usual HDL-induced inhibition of endothelial expression of vascular cell adhesion molecule 1 is impaired in patients with type 2 diabetes and CVD [55]. This anti-inflammatory activity of HDL is lost due to favoring the adhesion of macrophages to activated endothelial cells in patients with diabetes [56]. Moreover, it has been shown that the HDL paricle in this group of patients loses its vasorelaxant effects. It has also been confirmed that in this group of patients, HDL has a reduced ability to stimulate endothelial nitric oxide production and endothelial-dependent vasodilation [57].

Interestingly, Riwanto et al showed that HDL in patients with type 2 diabetes with CHD does not inhibit endothelial apoptosis because it fails to activate antiapoptotic proteins, while simultaneously stimulating proapoptotic pathways [58]. Furthermore, a study of diabetic patients showed that cholesterol efflux was positively associated with the total number of HDL-P but not with HDL-C or apoA-I level [59].

HDL and oxidative stress. In some clinical situations, high HDL-C level might be unfavourable. For example, in acute phase response, as seen after surgery, or in inflammation or diabetes, HDL may undergo some changes that affect its quality even though its level remains within the normal range [60-62].

Recent studies have focused on so-called dysfunctional HDL, which is HDL deprived of its primary function as well as atheroprotective and anti-inflammatory actions [63]. The latest observational studies have shown that the antiatherosclerotic effect of normally functioning HDL is frequently impaired in clinical states associated with systemic inflammation [63]. It is hypothesized that common atherosclerotic risk factors, such as dyslipidaemia, diabetes, hypertension, sedentary life style, obesity, cigarette smoking, or unhealthy diet, are all characterized by the presence of systemic inflammation and oxidative stress and may explain why HDL loses its protective features [64]. It has been also suggested that both systemic inflammation and oxidative stress involve the conversion of HDL to a dysfunctional form, which is no longer cardioprotective $[50,65,66]$.

The conversion of antiatherogenic HDL molecules to proatherogenic dysfunctional HDL is caused by the loss of activity of antioxidant enzymes, such as paraoxonase-1 (PON-1), and also by chemical modifications of apoproteins and enhancement of acute phase protein response [67]. 
It has been shown that in such conditions, acute phase proteins are the major protein component of HDL particles, constituting $27 \%$ of the whole composition. However, the mechanism underlying this transformation is still unclear, and there are no widely accepted methods for determining HDL function in selected groups of patients. It has been suggested that these transformed HDL particles with reduced antioxidative activity may become a more useful biomarker of cardiovascular risk than the 'old' HDL. The effects of HDL's conversion to its dysfunctional form are presented in Table 3.

Table 3. Effects of HDL-C conversion to its dysfunctional form

Loss of antioxidant activity

Chemical modifications of apoproteins

Enhancement of acute phase proteins

It is believed that antiatherogenic effects of the higher numbers of HDL-P may be related to protein or other cargo (e.g. apoA-I, PON-1, myeloperoxidase [MPO]), rather than to cholesterol cargo of HDL [45]. The main antioxidant protein in HDL is apoA-I, whose role strictly depends on MPO. Recent studies have revealed that deficiency in apoA-I plays a major role in the development of atherosclerotic lesions, and that there is a close relationship between high MPO level and the development of atherosclerotic plaque. High apoA-I level was shown to be associated with a reduced risk of atherosclerotic plaque and inhibition of atherosclerosis [68]. MPO is found in neutrophils and monocytes and plays an important role in killing microorganisms. MPO catalytically consumes nitric oxide in vitro and in vivo and promotes lipid peroxidation and activation of protease cascades involved in plaque fissuring or rupture. This enzyme also induces the expression of endothelial cell tissue factor, oxidative conversion of LDL, and modification of apoA-I, impairing its ability to promote cholesterol efflux [69]. Finally, circulating MPO level strongly correlates with the degree of endothelial dysfunction [70].

Recent studies have revealed the unfavourable effect of MPO in numerous clinical conditions associated with systemic inflammation and oxidative stress, such as type 2 diabetes [71]. It has been shown that the expression of MPO is markedly enhanced in human atherosclerotic lesions. It binds with apoA-I and modifies HDL-C. When apoA-I is oxidized by MPO, its ability to promote cellular cholesterol efflux by ABCA1 pathway is diminished [66]. In addition, biochemical studies have revealed that oxidation of specific tyrosine and methionine residues in apoA-I contributes to the loss of ABCA1 activity. Therefore, one potential pathway involves oxidative damage of HDL proteins by MPO. A mass spectrometric analysis demonstrated that the levels of 3-chlorotyrosine and 3-nitrotyrosine, two characteristic products of MPO, are elevated in HDL isolated from patients with established CVD. In summary, it may be hypothesize that plasma levels of MPO and its activity are higher in type 2 diabetes patients with CVD than in non-diabetic patients.

There are also other enzymes that can potentially modify HDL proteins. A number of studies have shown that the antioxidant activity of HDL is associated with the levels of PON-1 protein. PON-1 is an enzyme that is synthesized in the liver and is present in HDL particles consisting of apoA-I. PON1 slows down lipid oxidation and contributes to antioxidant and atheroprotective effects of HDL. Its protective properties result not only from the ability to eliminate potential oxidants but also from the ability to neutralize their final products to nontoxic particles. Recent studies have shown a low level of PON-1 in patients with type 2 diabetes [72]. Rani et al. reported a significant reduction in PON1 activity along with a decrease in HDL-C level in diabetic patients in comparison with nondiabetic individuals. Moreover, the authors stated that the progression of diabetes over the years resulted in a much higher reduction in PON-1 activity, as confirmed by Pearsons' correlation analysis. Thus, they concluded that diabetes, as a condition associated with oxidative stress, leads to a reduction in the antioxidant activity of PON-1. Also, lower serum level of PON-1 has been associated with higher morbidity and mortality related to cardiovascular complications in patients with type 2 diabetes. Taken together, the above findings suggest that PON-1 may be a better predictor of atherosclerotic risk in type 2 diabetes than HDL.

Some recent studies have demonstrated that plasma MPO level has a significant inverse correlation with PON-1 level in patients with stable and unstable angina pectoris, and suggested that the imbalance between pro-oxidants and antioxidants may contribute to the progression of coronary plaque instability [73]. Based on current knowledge, the authors of the current study believe that there may be such a correlation in patients with stable ischemic disease and type 2 diabetes, but this issue requires further studies.

HDL and malondialdehyde. Diabetes mellitus is a condition associated with oxidative stress. Extensive research has shown that an increased production of reactive oxygen species results in tissue injury in such pathological conditions as diabetes or chronic degenerative diseases [74]. Some recent studies have demonstrated that malondialdehyde (MDA) and phospholipid aldehydes can be produced under the condition of oxidative stress and contribute to tissue injury and dysfunction by depleting glutathione, leading to the modification of proteins, lipids, and DNA [74]. Recently, Shao et al. have shown that the exposure to increased concentrations of MDA progressively and dramatically deprived ApoA-I of its ability to promote cholesterol efflux $[75,76]$. Moreover, it has been reported that MDA levels are elevated in diseases associated with an increased risk of CVD, such as diabetes [77]. Importantly, immunochemical analyses demonstrated that HDL isolated from atherosclerotic tissue contained higher levels of MDA-modified proteins than HDL originating from the plasma of apparently healthy humans [76]. This suggests that MDA modifies HDL in human atherosclerotic tissue.

HDL and coagulation. Several studies have confirmed the anticoagulant properties of $\mathrm{HDL}-\mathrm{C}$, although there have also been studies suggesting that it has pro-coagulant effects [78-80]. This is due to the fact that HDL-C is a very heterogeneous group of particles. ApoA-I, the predominant protein of HDL-C, was shown to have antiatherogenic properties mostly associated with reverse cholesterol transport [81-83]. However apoA-I oxidation could modify its atheroprotective features $[78,84,85]$. On the other hand, apoA-II, the second major HDL particle, has been found to be associated with pro-coagulant effects and progression of atherosclerosis [86-88]. Of note, the EPIC-Norfolk study demonstrated that apoA-II was not associated with coronary 
artery disease and its major complication--myocardial infarction. Coagulant properties of HDL-C, and particularly their effects on platelet function, still remain unknown. A few studies showed that HDL particles change platelet signaling pathways by limiting intraplatelet cholesterol overload and inhibiting the production of nitric oxide and prostacyclin $[89,90]$. It is still not known whether HDL particles influence the tissue expression on the endothelial surface $[78,91,92]$. Nevertheless, decreased HDL-C level was associated with high platelet reactivity, both in patients with ST-segment elevation myocardial infarction and in those with stable coronary artery disease $[93,94]$.

Association of HDL with diet and physical activity. The effect of lifestyle interventions, such as the use of diet, on HDL function in patients with type 2 diabetes remains unclear [95]. However, for example, the traditional Mediterranean diet is now widely recommended in the prevention of CVD.

A recent meta-analysis reported that a lifestyle intervention resulted in a significant improvement of risk factors associated with CVD, such as body mass index, haemoglobin $\mathrm{A}_{1 \mathrm{c}}$, systolic blood pressure, and diastolic blood pressure, in patients with type 2 diabetes. However, HDL-C was not significantly changed by the intervention [96-98]. The described lifestyle intervention included an exercise and diet component and at least one other component such as smoking cessation, behavior modification, or counseling. A further analysis of the data revealed an improvement in HDL and haemoglobin $\mathrm{A}_{1 \mathrm{c}}$ levels only for interventions that included pharmacotherapy [93].

In contrast, a recent multicentre randomized clinical trial, Look AHEAD (Action for Health in Diabetes), showed a significant effect of an intensive lifestyle intervention on HDL-C in comparison with the control group [99]. This association was not shown for other risk factors. The intervention included diet modification and physical activity. In order to increase dietary compliance, a portion-controlled diet was used, with liquid meal replacements provided free of charge and recommendations to use other portioncontrolled items. In addition, the authors aimed to encourage the participants to achieve at least 175 minutes of physical activity per week, using activities similar in intensity to brisk walking. Behavioural strategies included self-monitoring. During each of the four years of this study, HDL-C level in the group of patients receiving the lifestyle intervention was approximately $8 \%-9 \%$ higher, compared with baseline levels. The results of another recent study confirmed that encouraging adults with newly diagnosed type 2 diabetes to interrupt prolonged periods of sedentary time with physical activity may be an effective strategy to maintain metabolic health [100]. The study showed that reallocation of 30 minutes of long-bout sedentary time with light physical activity was associated with higher HDL-C level.

To the knowledge of the authors of the presented study, there are only a few studies that analyzed the correlation between diet and apoA-I and apoA-II profiles in patients suffering from CHD and type 2 diabetes. Also, only a few studies have investigated gender-related differences in HDL-C level or apoA-I and apoA-II level in response to diet. Nevertheless, some evidence suggests that women could respond differently to diet compared with men, partly due to sex hormones [101, 102]. In fact, the response of HDL-C to alterations in dietary fats and carbohydrates appears to be greater in women than in men [101]. Another recent study has revealed a more pronounced decrease in apoA-II level in men than in women after introducing an experimental Mediterranean diet [103]. Moreover, the LDL-C to HDL-C ratio was also significantly decreased in men; therefore, the authors suggested that the decrease in apoA-II level in men in response to the Mediterranean diet did not affect the metabolism of plasma lipids and lipoproteins.

The above-results show that further studies are needed to improve our knowledge on the effect of diet and physical activity on HDL-C, apoA-I, and apoA-II level in patients with type 2 diabetes. This might allow the development of more specific nutritional strategies for the prevention of CVD.

\section{CONCLUSIONS}

Type 2 diabetes is currently considered a worldwide epidemic; therefore, it is crucial to find an effective therapeutic strategy for this disease. The progress in our knowledge about lipid disorders and their influence on the prognosis of patients with $\mathrm{CHD}$ and type 2 diabetes may soon require the development of a new and more specific lipid panel, as the one used in current medical practice may prove insufficient. The standard measurement of HDL-C level may not be enough to reflect its antiatherogenic potential because cardiovascular events have been widely observed in type 2 diabetes patients with normal or even increased HDL-C level. Therefore, the search for new markers with a better prognostic value is needed in this patient group.

The association between dysfunctional HDL and type 2 diabetes is important, both for physicians and for clinical researchers, but to-date the issue has not been fully elucidated. Currently, several studies investigating these relationships are being conducted. The entire March 2015 issue of the European Heart Journal focused on lipoproteins and diabetes [104]. The journal's Editor-in-Chief, Thomas Luscher, underlined that despite all the progress that had been made, there were still some unanswered questions concerning the involvement of lipids in CVD. In particular, he mentioned such issues as the management of homozygous autosomal dominant hypercholesterolaemia, the role of HDL-C, and the problem of obesity [105-107]. He also underlined that all attempts to modify HDL-C pharmacologically have failed so far [104].

All the data reviewed above lay the foundation for a new approach to understanding how the functional properties of HDL help reduce the risk of CVD. Therefore, in the light of those findings, there is a need to find a better diagnostic marker than HDL-C level. It is hoped that the elucidation of the mechanisms of oxidative stress and its effects on HDL in type 2 diabetes may take us a step further in the ongoing search for new lipid markers.

\section{REFERENCES}

1. International Diabetes Federation. http://www.diabetesatlas.org/ Accessed 14 Jun 2018.

2. Guariguata L, Whiting DR, Hambleton I, et al. Global estimates of diabetes prevalence for 2013 and projections for 2035. Diabetes Res Clin Pract. 2014; 103(2): 137-149.

3. Danaei G, Lawes CM, Vander Hoorn S, et al. Global and regional mortality from ischaemic heart disease and stroke attributable to higher-than-optimum blood glucose concentration: comparative risk assessment. Lancet 2006; 368(9548): 1651-1659. 
4. Lloyd-Jones D, Adams R, Carnethon M, et al. Heart disease and stroke statistics-2009 update: a report from the American Heart Association Statistics Committee and Stroke Statistics Subcommittee. Circulation 2009; 119(3): e21-181

5. Xiao C, Dash S, Morgantini C. Pharmacological Targeting of the Atherogenic Dyslipidemia Complex: The Next Frontier in CVD Prevention Beyond Lowering LDL Cholesterol. Diabetes. 2016; 65(7): 1767-78.

6. Ference BA, Ginsberg HN, Graham, et al. Low-density lipoproteins cause atherosclerotic cardiovascular disease. Evidence from genetic, epidemiologic, and clinical studies. A consensus statement from the European Atherosclerosis Society Consensus Panel. Eur Heart J. 2017; 38: 2459-2472

7. Ference BA, Yoo W, Alesh I et al. Effect of long-term exposure to lower low-density lipoprotein cholesterol beginning early in life on the risk of coronary heart disease: a Mendelian randomization analysis. J Am Coll Cardiol. 2012; 60: 2631-2639.

8. Ragbir S, Farmer JA. Dysfunctional High-Density Lipoprotein and Atherosclerosis. Curr. Atheroscler Rep. 2010; 12(5): 343-348.

9. Farmer JA, Liao J. Evolving concepts of the role of high-density lipoprotein in protection from atherosclerosis. Curr Atheroscler Rep. 2011; 13(2): 107-114.

10. Brewer Jr HB, Remaley AT, Neufeld EB, et al. Regulation of plasma highdensity lipoprotein levels by the ABCA1 transporter and the emerging role of high-density lipoprotein in the treatment of cardiovascular disease. Arterioscler Thromb Vasc Biol. 2004; 24: 1755-60.

11. Rigotti A, Miettinen HE, Krieger M. The role of the high-density lipoprotein receptor SR-BI in the lipid metabolism of endocrine and other tissues. Endocr Rev. 2003; 24: 357-87.

12. Castro G, Nihoul LP, Dengremont C, et al. Cholesterol efflux, lecithincholesterol acyltransferase activity, and pre-beta particle formation by serum from human apolipoprotein A-I and apolipoprotein A-I/ apolipoprotein A-II transgenic mice consistent with the latter being less effective for reverse cholesterol transport. Biochemistry 1997; 36(8): 2243-2249.

13. Asztalos BF, Roheim PS, Milani RL, et al. Distribution of apo A-Icontaining HDL subpopulations in patients with coronary heart disease. Arterioscler Thromb Vasc Biol. 2000; 20(12):2670-2676.

14. Chrysohoou C, Pitsavos C, Skoumas J, et al. The emerging antiinflammatory role of HDL-cholesterol, illustrated in cardiovascular disease free population; the ATTICA study. Int J Cardiol. 2007; 122(1): 29-33.

15. Castelli WP. Cholesterol and lipids in the risk of coronary artery disease-the Framingham Heart Study. Can J Cardiol. 1988; 4(Suppl. A): $5 \mathrm{~A}-10 \mathrm{~A}$.

16. Farmer JA, Liao J. Evolving concepts of the role of high-density lipoprotein in protection from atherosclerosis. Curr Atheroscler Rep. 2011; 13(2): 107-114.

17. Fielding CJ, Fielding PE. Molecular physiology of reverse cholesterol transport. J Lipid Res. 1995; 36(2): 211-228.

18. Phillips MC, Gillotte KL, Haynes MP, Johnson WJ, Lund-Katz S, Rothblat GH. Mechanisms of high density lipoprotein-mediated efflux of cholesterol from cell plasma membranes. Atherosclerosis 1998; 137(Suppl.): S13-17.

19. Baigent C, Keech A, Kearney PM, et al. Efficacy and safety of cholesterollowering treatment: prospective meta-analysis of data from 90,056 participants in 14 randomised trials of statins. Lancet $2005 ; 366(9493)$ : 1267-1278.

20. Farnier M. An evaluation of alirocumab for the treatment of hypercholesterolemia. Expert Rev Cardiovasc Ther. 2015; 13(12): 1307-1323.

21. Ridker PM, Hennekens CH, Buring JE, et al. C-reactive protein and other markers of inflammation in the prediction of cardiovascular disease in women. N Eng J Med. 2000; 342(12): 836-843.

22. HPS2-THRIVE Collaborative Group, Landray MJ, Haynes R, et al. Effects of extended-release niacin with laropiprant in high-risk patients. N Engl J Med. 2014; 371(3): 203-212.

23. HPS2-THRIVE Collaborative Group1. HPS2-THRIVE randomized placebo-controlled trial in 25673 high-risk patients of ER niacin/ laropiprant: trial design, pre-specified muscle and liver outcomes, and reasons for stopping study treatment. Eur Heart J. 2013; 34(17): 1279-1291.

24. Mooradian AD, Haas MJ. Targeting high-density lipoproteins: increasing de novo production versus decreasing clearance. Drugs 2015; 75(7): 713-722.

25. Barter P. Lessons learned from the Investigation of Lipid Level Management to Understand its Impact in Atherosclerotic Events (ILLUMINATE) trial. Am J Cardiol. 2009; 104: 10E-15E.
26. Bots ML, Visseren FL, Evans GW, et al. Torcetrapib and carotid intimamedia thickness in mixed dyslipidaemia (RADIANCE 2 study): a randomised, double-blind trial. Lancet 2007; 370: 153-60.

27. Schwartz GG, Olsson AG, Abt M, et al. Effects of dalcetrapib in patients with a recent acute coronary syndrome. N Engl J Med. 2012; 367: 2089-99.

28. Hovingh GK, Kastelein J, van Deventer SJ, et al. Cholesterol ester transfer protein inhibition by TA-8995 in patients with mild dyslipidaemia (TULIP): a randomised, double-blind, placebo-controlled phase 2 trial. Lancet. 2015; 386(9992): 452-60.

29. Keene D, Price C, Shun-Shin MJ, et al. Effect on cardiovascular risk of high density lipoprotein targeted drug treatments niacin, fibrates, and CETP inhibitors: meta-analysis of randomised controlled trials including 117,411 patients. BMJ Br Med J. 2014; 349: g4379.

30. Teslovich TM, Musunuru K, Smith AV, et al. Biological, clinical and population relevance of 95 loci for blood lipids. Nature. 2010; 466: 707-713.

31. Manolio TA, Collins FS, Cox NJ, et al. Finding the missing heritability of complex diseases. Nature. 2009; 461: 747-753.

32. Voight BF, Peloso GM, Orho-Melander M, et al. Large-scale genecentric meta-analysis across 32 studies identifies multiple lipid loci. Am J Hum Genet. 2012; 91: 823-838.

33. Holmes MV, Asselbergs FW, Palmer TM, et al. Mendelian randomization of blood lipids for coronary heart disease. Eur Heart J. 2015; 36: 539-550.

34. Boekholdt SM, Arsenault BJ, Hovingh GK, et al. Levels and changes of HDL cholesterol and apolipoprotein A-I in relation to risk of cardiovascular events among statin-treated patients: a meta-analysis. Circulation. 2013; 128(14): 1504-12.

35. Ray K, Wainwright NW, Visser L, et al. Changes in HDL cholesterol and cardiovascular outcomes after lipid modification therapy. Heart. 2012; 98: 780-785.

36. Briel M, Ferreira-Gonzalez I, You JJ, et al. Association between change in high density lipoprotein cholesterol and cardiovascular disease morbidity and mortality: systematic review and meta-regression analysis. BMJ. 2009; 16: 338:b92.

37. Grover SA, Kaouache M, Joseph L, et al. Evaluating the incremental benefits of raising high-density lipoprotein cholesterol levels during lipid therapy after adjustment for the reductions in other blood lipid levels. Arch Intern Med. 2009; 169(19): 1775-80.

38. Boden WE, Probstfield JL, Anderson T, et al. Niacin in patients with low HDL cholesterol levels receiving intensive statin therapy. N Engl J Med. 2011; 365(24): 2255-67.

39. Nissen SE, Tardif JC, Nicholls SJ, et al. Effect of torcetrapib on the progression of coronary atherosclerosis. N Engl J Med. 2007; 356(13): 1304-16.

40. Barter PJ, Caulfield M, Eriksson M, et al. Effects of torcetrapib in patients at high risk for coronary events. N Engl J Med. 2007; 357: 2109-2122.

41. Asztalos BF, Tani M, Schaefer EJ. Metabolic and functional relevance of HDL subspecies. Curr Opin Lipidol. 2011; 22(3): 176-85.

42. Mora S, Glynn RJ, Ridker PM. High-density lipoprotein cholesterol, size, particle number, and residual vascular risk after potent statin therapy. Circulation. 2013; 128(11): 1189-97.

43. Vergeer M, Holleboom AG, Kastelein JJ. The HDL hypothesis: does high-density lipoprotein protect from atherosclerosis? J Lipid Res. 2010; 51(8): 2058-73.

44. Mackey RH, Greenland P, Goff DC Jr, et al. High-density lipoprotein cholesterol and particle concentrations, carotid atherosclerosis, and coronary events: MESA (multi-ethnic study of atherosclerosis). J Am Coll Cardiol. 2012; 60(6): 508-16.

45. deGoma EM, deGoma RL, Rader DJ. Beyond high-density lipoprotein cholesterol levels evaluating high-density lipoprotein function as influenced by novel therapeutic approaches. J Am Coll Cardiol. 2008; 51(23): 2199-211.

46. Rader DJ, Alexander ET, Weibel GL. The role of reverse cholesterol transport in animals and humans and relationship to atherosclerosis. J Lipid Res. 2009; 50: S189-94.

47. Saddar S, Carriere V, Lee WR, et al. Scavenger receptor class B type I is a plasma membrane cholesterol sensor. Circ Res. 2013; 112(1): 140-51.

48. Murphy AJ, Bijl N, Yvan-Charvet L, et al. Cholesterol efflux in megakaryocyte progenitors suppresses platelet production and thrombocytosis. Nat Med. 2013; 19(5): 586-94.

49. Tall AR. Cholesterol efflux pathways and other potential mechanisms involved in the athero-protective effect of high density lipoproteins. J Intern Med. 2008; 263(3): 256-73.

50. Khera AV, Cuchel M, de la Llera-Moya M, et al. Cholesterol efflux capacity, high-density lipoprotein function, and atherosclerosis. N Engl J Med. 2011; 364(2): 127-135. 
51. Rohatgi A, Khera A, Berry JD, et al. HDL cholesterol efflux capacity and incident cardiovascular events. N Engl J Med. 2014; 371(25): 2383-93.

52. Hutchins PM, Heinecke JW. Cholesterol efflux capacity, macrophage reverse cholesterol transport and cardioprotective HDL. Curr Opin Lipidol. 2015; 26(5): 388-93.

53. Cuchel M, Rader DJ. Macrophage reverse cholesterol transport: key to the regression of atherosclerosis? Circulation. 2006; 113(21): 2548-55.

54. Sorci-Thomas MG, Bhat S, Thomas MJ. Activation of lecithin: cholesterol acyltransferase by HDL ApoA-I central helices. Clin Lipidol. 2009; 4(1): 113-124.

55. Hoang A, Murphy AJ, Coughlan MT, et al. Advanced glycation of apolipoprotein A-I impairs its anti-atherogenic properties. Diabetologia 2007; 50(8): 1770- 1779 .

56. Ansell BJ, Navab M, Hama S,. et al. Inflammatory/anti-inflammatory properties of high density lipoprotein distinguish patients from control subjects better than high-density lipoprotein cholesterol levels and are favorably affected by simvastatin treatment. Circulation. 2003; 108: 2751-6.

57. Sorrentino SA, Besler C, Rohrer L, et al. Endothelial-vasoprotective effects of high density lipoprotein are impaired in patients with type 2 diabetes mellitus but are improved after extended-release niacin therapy. Circulation 2010; 121(1): 110-122.

58. Riwanto M, Rohrer L, Roschitzki B, et al. Altered activation of endothelial anti- and proapoptotic pathways by high-density lipoprotein from patients with coronary artery disease: role of high-density lipoprotein proteome remodeling. Circulation 2013; 127(8): 891-904.

59. Tan HC, Tai ES, Sviridov D, et al. Relationships between cholesterol efflux and high-density lipoprotein particles in patients with type 2 diabetes mellitus. J Clin Lipidol. 2011; 5(6): 467-73.

60. Smith J. Myeloperoxidase, inflammation, and dysfunctional HDL. J. Clin. Lipidol. 2010; 4(5): 382-388.

61. Navab M, Anantharamaiah G, Fogelman A. The role of High-Density Lipoprotein in Inflammation. Trends. Cardiovasc Med. 2005; 15(4): $158-161$.

62. Morgantini C, Natali A, Boldrini B, et al. Anti-inflammatory and Antioxidant Properies of HDLs are impaired in Type 2 Diabetes. Diabetes 2011; 60(10): 2617-2623.

63. Ragbir S, Farmer JA. Dysfunctional High-Density Lipoprotein and Atherosclerosis. Curr Atheroscler Rep. 2010; 12(5): 343-348.

64. Van Lenten BJ, Hama SY, de Beer FC, et al. Anti-inflammatory HDL becomes pro-inflammatory during the acute phase response. Loss of protective effect of HDL against LDL oxidation in aortic wall cell cocultures. J Clin Invest. 1995; 96(6): 2758-2767.

65. Barter PJ, Nicholls S, Rye KA, et al. Antiinflammatory properties of HDL. Circ Res. 2004; 95(8): 764-772.

66. Shao B, Oda MN, Oram JF, et al. Myeloperoxidase: an oxidative pathway for generating dysfunctional high-density lipoprotein. Chem Res Toxicol. 2010; 23(3): 447-454.

67. Patra SK, Singh K, Singh R. Paraoxonase 1: A better atherosclerotic risk predictor than HDL in type 2 diabetes mellitus. Diabetes Metab Syndr. 2013; 7(2): 108-111.

68. Podrez EA. Anti-oxidant properties of high-density lipoprotein and atherosclerosis. Clin Exp Pharmacol Physiol. 2010; 37(7): 719-25.

69. Zheng L, Nukuna B, Brennan ML, et al. Apolipoprotein A-I is a selective target for myeloperoxidase-catalyzed oxidation and functional impairment in subjects with cardiovascular disease. J Clin Invest. 2004; 114(4): 529-41.

70. Shao B, Pennathur S, Heinecke JW. Myeloperoxidase Targets Apolipoprotein A-I, the Major High Density Lipoprotein Protein, for Site-Specific Oxidation in Human Atherosclerotic Lesions. J Biol Chem. 2012; 287(9): 6375-6386.

71. Song P, Xu J, Song Y, et al. Association of Plasma Myeloperoxidase Level with Risk of Coronary Artery Disease in Patients with Type 2 Diabetes. Dis Markers. 2015; 2015: 761939.

72. Jamuna Rani A, Mythili SV, Nagarajan S. Study on paraoxonase 1 in type 2 diabetes mellitus. Indian J Physiol Pharmacol. 2014; 58(1): 13-16.

73. Yunoki K, Naruko T, Inaba M, et al. Gender-specific correlation between plasma myeloperoxidase levels and serum high-density lipoproteinassociated paraoxonase- 1 levels in patients with stable and unstable coronary artery disease. Atherosclerosis 2013; 231(2): 308-314.

74. Singh M, Kapoor A, Bhatnagar A. Oxidative and reductive metabolism of lipid-peroxidation derived carbonyls. Chem Biol Interact. 2015; 234: $261-273$.

75. Shao B, Pennathur S, Pagani I, et al. Modifying Apolipoprotein A-I by Malondialdehyde, but Not by an Array of Other Reactive Carbonyls, Blocks Cholesterol Efflux by the ABCA1 Pathway. J Biol Chem. 2010; 285(24): 18473-18484.
76. Shao B. Site-specific oxidation of apolipoprotein A-I impairs cholesterol export by ABCA1, a key cardioprotective function of HDL. Biochim Biophys Acta. 2012; 1821(3): 490-501.

77. Slatter DA, Bolton CH, Bailey AJ. The importance of lipid-derived malondialdehyde in diabetes mellitus. Diabetologia 2000; 43(5): 550-557.

78. Viswambharan H, Ming XF, Zhu S, et al. Reconstituted highdensity lipoprotein inhibits thrombin-induced endothelial tissue factor expression through inhibition of RhoA and stimulation of phosphatidylinositol 3-kinase but not Akt/endothelial nitric oxide synthase. Circ Res. 2004; 94(7): 918-25.

79. Mineo C, Deguchi H, Griffin JH, et al. Endothelial and antithrombotic actions of HDL. Circ Res. 2006; 98(11): 1352-64.

80. Zimman A, Podrez EA. Regulation of platelet function by class B scavenger receptors in hyperlipidemia. Arteriosclerosis Thromb Vasc Biol. 2010; 30(12): 2350-6.

81. Moore RE, Navab M, Millar JS, et al. Increased atherosclerosis in mice lacking apolipoprotein A-I attributable to both impaired reverse cholesterol transport and increased inflammation. Circ Res. 2005; 97(8): 763-71.

82. Marcel YL, Kiss RS. Structure-function relationships of apolipoprotein A-I: a flexible protein with dynamic lipid associations. Curr Opin Lipidol. 2003; 14(2): 151-7.

83. Nicholls SJ, Zheng L, Hazen SL, et al. Formation of dysfunctional high-density lipoprotein by myeloperoxidase. Trends Cardiovasc Med. 2005; 15(6): 212-9.

84. Gotto AM Jr, Whitney E, Stein EA, et al. Relation between baseline and on-treatment lipid parameters and first acute major coronary events in the Air Force/Texas Coronary Atherosclerosis Prevention Study (AFCAPS/TexCAPS). Circulation. 2000; 101(5): 477-84.

85. Peng DQ, Brubaker G, Wu Z, et al. Apolipoprotein A-I tryptophan substitution leads to resistance to myeloperoxidase-mediated loss of function. Arterioscler Thromb Vasc Biol. 2008; 28(11): 2063-70.

86. Durbin DM, Jonas A. Lipid-free apolipoproteins A-I and A-II promote remodeling of reconstituted high density lipoproteins and alter their reactivity with lecithin: cholesterol acyltransferase. J Lipid Res. 1999; 40: 2293-2302.

87. Lagrost L, Perségol L, Lallemant C, et al. Influence of apolipoprotein composition of high density lipoprotein particles on cholesterol ester transfer protein activity. J Biol Chem. 1994; 269: 3189-3197.

88. Castellani LW, Navab M, Lenten BJ, et al. Overexpression of apolipoprotein AII in transgenic mice converts high density lipoproteins to proinflammatory particles. J Clin Invest. 1997; 100: 464-474.

89. Valiyaveettil M, Kar N, Ashraf MZ, et al. Oxidized high- density lipoprotein inhibits platelet activation and aggregation via scavenger receptor BI. Blood. 2008; 111: 1962-1971.

90. Caughey GE, Cleland LG, Penglis PS, et al. Roles of cyclooxygenase (COX)-1 and COX-2 in prostanoidproduction by humanendothelialcells: selectiveup-regulation of prostacyclinsynthesis by COX-2. J Immunol. 2001; 167: 2831 - 2838.

91. Mineo C, Deguchi H, Griffin JH, et al. Endothelial and antithrombotic actions of HDL. Circ Res. 2006; 98: 1352-1364.

92. Rosenson RS. Functional assessment of HDL: Moving beyond static measures for risk assessment. Cardiovasc Drugs Ther. 2010; 24(1): 71-5.

93. Schäfer A, Flierl U, Kössler J, et al. Early determination of clopidogrel responsiveness by platelet reactivity indexidentifies patients at risk for cardiovascular events after myocardial infarction. Thromb Haemost. 2011; 106(1): 141-8.

94. Schäfer A, Weinberger S, Flierl U, et al. ADP-induced platelet aggregation frequently fails to detect impaired clopidogrel-responsiveness in patients with coronary artery disease compared to a P2Y12-specific assay. Thromb Haemost. 2008; 100(4): 618-25.

95. Chen L, Pei JH, Kuang J, et al. Effect of lifestyle intervention in patients with type 2 diabetes: a meta-analysis. Metabolism 2015; 64(2): 338-47.

96. Angermayr L, Melchart D, Linde K. Multifactoriallifestyle interventions in the primary and secondary prevention of cardiovascular disease and type 2 diabetes mellitus-a systematic review of randomized controlled trials. Ann Behav Med 2010; 40: 49-64.

97. Schellenberg ES, Dryden DM, Vandermeer B, et al. Lifestyle interventions for patients with and at risk for type 2 diabetes: a systematic review and meta-analysis. Ann Intern Med. 2013; 159: 543-51.

98. Yang Z, Scott CA, Mao C, et al. Resistance exercise versus aerobic exercise for type 2 diabetes: a systematic review and meta-analysis. Sports Med. 2014; 44(4): 487-99.

99. Look AHEAD Research Group, Wing R.R. Long-term effects of a lifestyle intervention on weight and cardiovascular risk factors in individuals with type 2 diabetes mellitus: four-year results of the Look AHEAD trial. Arch Intern Med. 2010; 170(17): 1566-75. 
100. Falconer CL, Page AS, Andrews RC, et al. The Potential Impact of Displacing Sedentary Time in Adults with Type 2 Diabetes. Med Sci Sports Exerc. 2015; 47(10): 2070-5.

101. Knopp RH, Paramsothy P, Retzlaff BM, et al. Gender differences in lipoprotein metabolism and dietary response: basis in hormonal differences and implications for cardiovascular disease. Curr Atheroscler Rep. 2005; 7(6): 472-9.

102. Lapointe A, Balk EM, Lichtenstein AH. Gender differences in plasma lipid response to dietary fat. Nutr Rev. 2006; 64(5): 234-49.

103. Bédard A, Riverin M, Dodin S, et al. Sex differences in the impact of the Mediterranean diet on cardiovascular risk profile. Br J Nutr. 2012; 108(8): 1428-34.

104. Lüscher TF. Lipoproteins and diabetes. Eur Heart J. 2015; 36(9): 529-31.
105. Cuchel M, Bruckert E, Ginsberg HN, et al. European Atherosclerosis Society Consensus Panel on Familial Hypercholesterolaemia. Homozygous familial hypercholesterolaemia: new insights and guidance for clinicians to improve detection and clinical management. A position paper from the Consensus Panel on Familial Hypercholesterolaemia of the European Atherosclerosis Society. Eur Heart J. 2014; 35: 2146-2157.

106.Luscher TF, Taddei S, Kaski JC, et al. Vascular effects and safety of dalcetrapib in patients with or at risk of coronary heart disease: the dal-VESSEL randomized clinical trial. Eur Heart J. 2012; 33: 857-865.

107. Simic B, Hermann M, Shaw SG, et al. Torcetrapib impairs endothelial function in hypertension. Eur Heart J. 2012; 33: 1615-1624. 